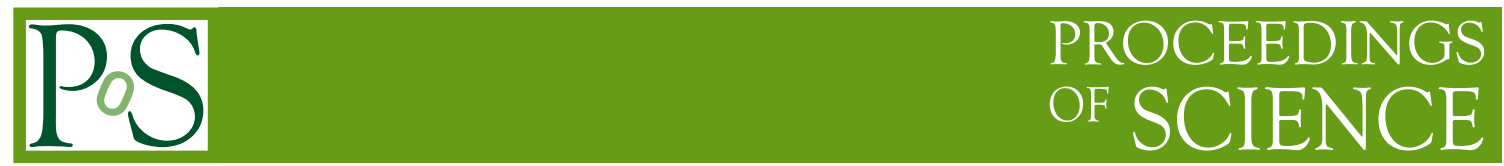

\title{
New Perspectives in Multispectral Sky Monitoring
}

\author{
René Hudec ${ }^{1,2 * \dagger}$ \\ ${ }^{1}$ Czech Technical University in Prague, Faculty of Electrical Engineering, \\ Technicka 2, CZ 16000 Prague, Czech Republic \\ ${ }^{2}$ Astronomical Institute of the Academy of Sciences of the Czech Republic \\ Fricova 298 - CZ 25165 Ondrejov, Czech Republic \\ E-mail: rene.hudeclgmail.com
}

I give a short review and discussion of multispectral (optical and X-rays) sky monitoring.

XI Multifrequency Behaviour of High Energy Cosmic Sources Workshop

25-30 May 2015

Palermo, Italy

* Speaker.

${ }^{\dagger}$ A footnote may follow. 


\section{Introduction}

Many objects discussed at this workshop (XI Multifrequency Behaviour of High Energy Cosmic Sources Workshop) are (mostly variable) emitters of optical light and of X rays, hence mutispectral sky monitoring is very important to understand their physical nature.

\section{Optical Sky Monitoring and Sky Patrol}

Here we understand optical sky patrol as extended database, covering large sky area (in ideal case full either northern or southern sky hemisphere) over long time interval (years or more) with reasonably dense sampling (1 point per week or better).

Despite many optical surveys in past or recent operation, very few fulfill this condition. The main motivation of multispectral sky monitoring is as follows: study of long term optical behavior of high-energy (but also other) sources, monitoring of rare eruptive phenomena and providing database for simultaneous and quasi simultaneous multifrequency observations.

Note that the term SURVEY is used mostly for deep but very infrequent monitoring.

\subsection{Photographic patrol surveys}

Photographic sky patrol was performed at many observatories mostly in the time interval 19001990 (Hudec, 2014). Recently there are very few photographic patrols left (i.e. meteor sky patrol at the Ondrejov Observatory). Nowadays the (historical) photographic surveys are digitized and they will be available online. One of first examples is the German APPLAUSE. But most of the data in astronomical photographic data archives (more than 7 millions of negatives) still remain invisible to professional astronomers, if digitized then the community would have 100 years coverage with thousand of points for every sky object. Despite introducing inexpensive ( 0.20 Euro per plate) and fast (2 sec) scanning technology (Hudec and Hudec, 2014), there is still problem with funding.

The APPLAUSE Collaboration has presented the first data release (DR1) of the APPLAUSE plate archives (Heber et al., 2014). The release contains data from the Bamberg, Hamburg and Potsdam plate collections. DR1 makes publicly available 25612 scans of 19335 plates and 1.66 billion extracted sources with positions and raw magnitudes. Plates in DR1 cover $98.9 \%$ of the entire sky. The oldest published plates date back to 1909 and the newest are from 1976. Additionally, there are 26526 digital images of plate envelopes and logbook pages from 77 logbooks in the release. APPLAUSE DR1 can be accessed at https://www.plate-archive.org/applause/

There are two large US archives with possible major impact on frontier astrophysics and dense sampling, namely (i) Baker Super Schmidt camera films 55 deg dia FOV, lim mag 15, dense sampling $20 \mathrm{sec}, \sim 110000$ negatives, $\sim 10$ years coverage, and (ii) Baker-Nunn camera networks negatives with FOV 30 x 5 deg, lim mag 16, very dense sampling $\sim$ few sec, more than 1 mil negatives, $\sim 20$ years coverage (Hudec and Hudec, 2014).

In addition to numerous photographic sky surveys, there are also extended photographic sky archives which focus on selected sky areas, and observations of selected objects such as novae and $\mathrm{SNe}$, eg. photographic archive at the Asiago Observatory (Fig. 1). 


\subsection{CCD patrol surveys}

There are very numerous past and recent CCD based optical sky patrol/sky survey programs. However many of them are specific for special purposes. Many of them represent narrow field surveys. Only few of them able to provide dense coverage for all objects on the sky. Few selected examples are listed below.

\subsubsection{ROTSE NSVS Sky Patrol}

The Northern Sky Variability Survey (NSVS) is a temporal record of the sky over the optical magnitude range from 8 to 15.5. It was conducted in the course of the first-generation Robotic Optical Transient Search Experiment (ROTSE-I) using a robotic system of four comounted unfiltered telephoto lenses equipped with CCD cameras. The survey was conducted from Los Alamos, New Mexico, and primarily covers the entire northern sky. Some data in southern fields between declinations 0 and -38 degrees are also available, although with fewer epochs and noticeably lesser quality. The NSVS contains light curves for approximately 14 million objects. With a 1 yr baseline and typically 100-500 measurements per object, the NSVS is the most extensive record of stellar variability across the bright sky available today.

ROTSE-I (Akerlof et al. 2000) monitored the entire observable sky twice a night. The Northern Sky Variability Survey (NSVS;Wozniak et al. 2004) is based on ROTSE-I data, with 100-500 measurements per object collected over one year. Typical photometric precision of this dataset is about 0.02 mag for sources not limited by photon statistics. ROTSE sky patrol was operated in the years 1999-2000. On a typical night, two patrol sequences were obtained, covering about 18000 deg2 of sky and recording the brightness of approximately $9 \times 10^{6}$ stars with four images taken in two epochs.

\subsubsection{ASAS}

The All Sky Automated Survey (ASAS; Pojmanski 2002) monitors the entire southern and part of the northern sky ( $\delta \leq+28$ degrees) to a limit of $\mathrm{V}=14$, including about 15 million stars. Typical photometric precision is about $0.05 \mathrm{mag}$. The third release of the ASAS Catalog of Variable Stars contains close to 50000 variable stars. About $80 \%$ of these are new discoveries (not previously cataloged). This program started in 1997.

\subsubsection{LONEOS}

The Lowell Observatory Near Earth Objects Survey Phase I (LONEOS-I; Miceli et al. 2008) provides photometric data for $1430 \mathrm{deg}^{2}$ of northern sky that has been imaged at least 28 times between 1998 and 2000. The LONEOS-I camera used no bandpass filter and reached a depth of R $\sim 18.5$. Typical photometric precision of this dataset is about 0.02 mag for sources not limited by photon statistics.

\subsubsection{Palomar Transient Factory}

The Palomar Transient Factory (PTF; Law et al. 2009 and Rau et al. 2009) is an ongoing wide-area, two-band (SDSS-g and Mould-R filters), deep $(\mathrm{R} \sim 20.6, \mathrm{~g} \sim 21.3$ ) survey aimed at systematic exploration of the optical transient sky. As of Fall 2011, PTF has observed about 7300 
$\operatorname{deg} 2$ of northern sky at least 30 times in the Mould-R band ( $\sim 1800 \operatorname{deg} 2$ of sky with more than 100 epochs). Typical photometric precision of this dataset is better than 0.01 for sources not limited by photon statistics.

\subsubsection{Pi of the Sky}

The Pi of the Sky experiment is located at INTA (Spain) and is represented by 4 mounts with 4 cameras that might work in coincidence or not. Each camera has FOV: 20 deg x 20 deg with limiting brightness $12 \mathrm{mag}$ and exposition time $10 \mathrm{~s}$. There are 2 cameras working in coincidence in San Pedro de Atacama (Chile), each camera with FOV: 20 deg x 20 deg, limiting brightness: 12 mag, and exposition time $10 \mathrm{~s}$ (Cwiek et al., 2014).

\section{Gaia}

The ESA Gaia satellite (e.g. Prusti, 2012) is designed to provide astrometry (V $\leq 20)$ : completeness to $20 \mathrm{mag}$ (on-board detection) with accuracy of $10^{-25}$ microarcsec at 15 mag (Hipparcos: 1 milliarcsec at $9 \mathrm{mag}$ ). Gaia is scanning satellite, with two viewing directions. Photometry ( $\mathrm{V} \leq$ 20 ) is performed as well for astrophysical diagnostics (low-dispersion photometry) + chromaticity.

\section{X-ray Sky Surveys}

Most of past and recent X-ray telescopes were with narrow field FOV (less than 1 degree diameter). Past X-ray surveys were based mostly on non imaging experiments (without use of optics) hence were of limited sensitivity.

\subsection{Lobster-Eye (LE)}

Lobster Eye Telescopes represent novel Wide Field X-ray Telescopes with FOV of 100 sq. deg. and more easily possible (classical X-ray optics has only 1 deg or less). They represent analogy with lobster eyes and were designed for astronomy, but laboratory applications are also possible. The Lobster Eye (LE) X-ray optics was originally proposed by Schmidt (1975) and Angel (1979). Since then, numerous test specimens of Lobster Eye telescopes were designed and tested (e.g. Inneman et al. 1999; Hudec et al. 2000, 2003, 2004; Tichý et al. 2009, 2011). The Lobster-Eye (LE) X-ray telescope can be miniaturized for an application in picosatellites. The LE telescopes are based on a real analogy with the lobster eyes.

The LE telescopes can typically serve in two basic operation modes as follows.

- Starrying (pointed) mode - only for satellite with pointing

- Scanning mode (no satellite pointing and/or stabilization required)

Parameters of LE optics are as follows. Energy range from optical to $\sim 10 \mathrm{keV}$ in $2 \mathrm{D}$ and from optical to $\sim 30 \mathrm{keV}$ in $1 \mathrm{D}$ mode, FOV typical 5x5 deg 1 module, more modules larger FOV, angular resolution $\sim 1$ to 10 arcmin typical, gain $100-1000$. Application areas are as follows. $\mathrm{X}$ ray astronomy in scanning mode, sky monitoring for transients, $\mathrm{X}$ ray astronomy in pointed mode, dense long term monitoring of selected sky area, XRF planetary science, X ray 
imaging in the laboratory, $\mathrm{X}$ ray laboratory collimators, $\mathrm{X}$ ray imaging of laboratory plasma, $\mathrm{X}$ ray security screeing and material research, $\mathrm{X}$ ray imaging and low resolution spectroscopy of triggers in atmosphere (balloon experiments), and $\mathrm{X}$ ray imaging and low resolution spectroscopy of polar lights (balloon and rocket experiments).

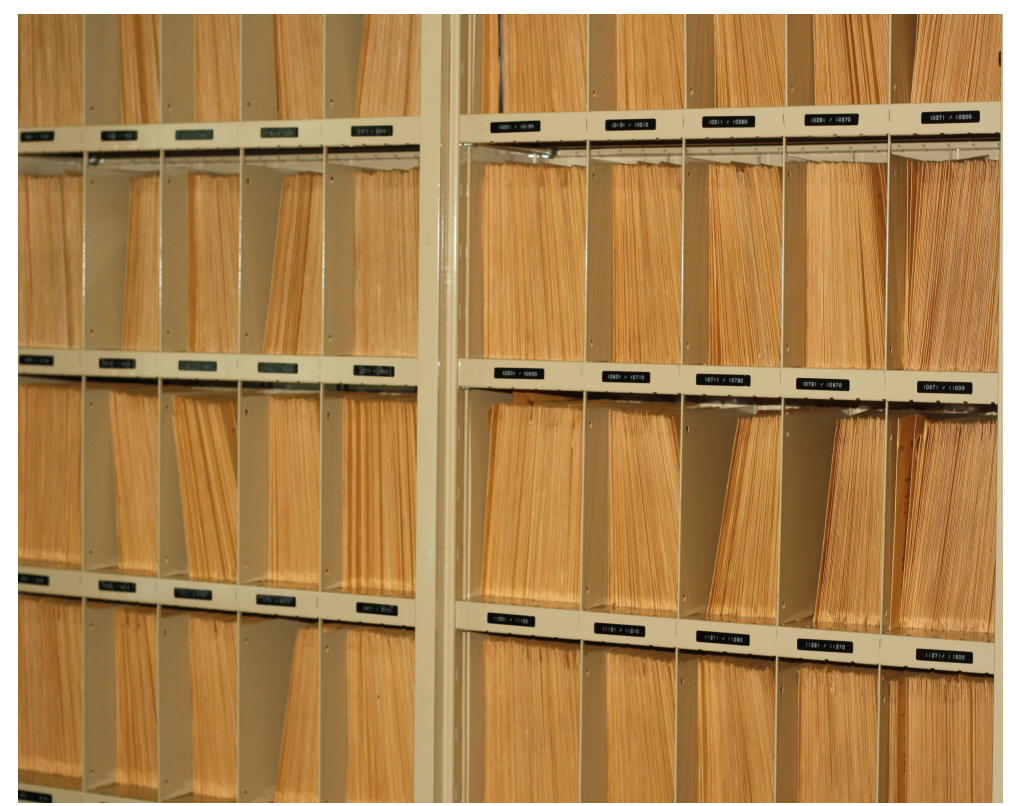

Figure 1: Photographic Archive at the Asiago Observatory, Italy)

\subsection{Science objectives of $X$-ray monitoring}

Wide field X-ray monitors of Lobster Eye type were demonstrated to play an important role in modern astrophysics (e.g. Hudec et al. 2007; Švéda et al. 2004). The most important scientific cases for monitor directed towards the Galaxy center are briefly summarized below.

- A long-term (months) measurement of the light curves of bright persistent X-ray binaries in the direction toward the center of the Galaxy in the soft X-ray band

- Detection and measurement of the light curves of bright transient events of X-ray binaries in the direction toward the center of the Galaxy in the soft X-ray band

\section{Conclusions}

The photographic sky patrols represent very important data base able to provide data over very long time intervals. The recent CCD surveys are numerous and very promising but the time coverage and/or sky coverage is mostly limited. For X-ray monitoring, monitors with imaging optics represent promising novel approach.

\section{Acknowledgments}

This work was supported by the grant GA CR 13-33324S (X-ray surveys) and by the GA CR grant 13-39464J as well (photographic optical surveys). 


\section{References}

[1] Akerlof, C. et al., The Astronomical Journal, Volume 119, Issue 4, pp. 1901-1913, 2000

[2] Cwiek A. et al., Proc. SPIE 9290, Photonics Applications in Astronomy, Communications, Industry, and High-Energy Physics Experiments 2014, 92900T (November 25, 2014); doi:10.1117/12.2076052

[3] Wozniak, P. R. et al., The Astronomical Journal, Volume 127, Issue 4, pp. 2436-2449, 2004

[4] Heber, U. et al., Astroplate 2014, Proceedings of a conference held in March, 2014 in Prague, Czech Republic. Edited by Linda Miskova and Stanislav Vitek. Published by the Institute of Chemical Technology, Prague, available online at http://astroplate.cz, 2014.

[5] Hudec, R., et al., Proc. SPIE 5488, UV and Gamma-Ray Space Telescope Systems, (11 October 2004); doi: 10.1117/12.551915, 2004

[6] Hudec, R., Pína, L., Inneman, A., Švéda, L., LOBSTER - Astrophysics with Lobster Eye Telescopes, in Exploring the Cosmic Frontier, ESO Astrophysics Symposia European Southern Observatory 2007, pp.73-74, 2007

[7] Hudec, R., Astroplate 2014, Proceedings of a conference held in March, 2014 in Prague, Czech Republic. Edited by Linda Miskova and Stanislav Vitek. Published by the Institute of Chemical Technology, Prague, available online at http://astroplate.cz, 2014, p.1

[8] Angel, J. R. P., 1979, Astroph. J., 364, 233

[9] Inneman, A., et al., 2000, Proc. SPIE, 4138, 94

[10] Hudec, R., et al., 2000, SPIE Proc. 4012, 432

[11] Hudec, R., et al., 2003, SPIE Proc. 4851, 578

[12] Hudec, R., et al., 2004a, SPIE Proc. 5488, 449

[13] Hudec, R., et al., 2004b, Nucl. Phys. B Proc. Suppl. 132, 320

[14] Hudec, R. and Hudec, L.,Acta Polytechnica CTU Proceedings 1(1), p.316-319, 2014.

[15] Hudec, L. and Hudec, R., Proceedings ASTROPLATE workshop, available online at http://astroplate.cz, 2015.

[16] Miceli A. et al., Miceli, A. et al. Astrophys.J. 678, 2008.

[17] Pojmanski, Acta Astronomica, v.52, pp.397-427, (2002)

[18] Law N. M. et al., Publications of the Astronomical Society of the Pacific, Volume 121, issue 886, pp.1395-1408, 2009

[19] Prusti, T., Astronomische Nachrichten, Vol.333, Issue 5-6, p.453, 2012.

[20] Rau, A. et al. Publications of the Astronomical Society of the Pacific, Volume 121, issue 886, pp.1334-1351, 2009

[21] Schmidt, M., Ravandoor, K., Kurz, O., Busch, S., Schilling, K., Attitude Determination for the Pico-Satellite UWE-2. In: Space Technology 28. 2009, pp.67-74, 2009

[22] Schmidt, W. K. H., 1975, NucIM, 127, 285

[23] Švéda, L., et al., 2004, SPIE Proc. 5168, 393

[24] Tichý, V., et al., 2009, Balt. Astr. 18, 362 
[25] Tichý, V., et al., 2011, Nucl. Instr. Meth. A, A633, S169

[26] Tichý, V., et al., 2013a, SPIE Proc. 8777, 877711

[27] Tichý, V., 2013b, SPIE Proc. 8777, 877710

[28] Hudec, R., et al., 2012, Acta Polytechnica, IBWS2011 Proceedings, 1(52) 